\title{
A Novel Quadrature-Tracking Demodulator for LTE-A Applications
}

\author{
Kang-Chun Peng $(\mathbb{D}$ and Chan-Hung Lee \\ Department of Computer and Communication Engineering, National Kaohsiung First University of Science and Technology, \\ 2 Jhuoyue Rd., Nanzih, Kaohsiung City 811, Taiwan
}

Correspondence should be addressed to Kang-Chun Peng; peterpkg@nkfust.edu.tw

Received 27 July 2017; Accepted 2 December 2017; Published 2 January 2018

Academic Editor: Chaojiang Li

Copyright (C) 2018 Kang-Chun Peng and Chan-Hung Lee. This is an open access article distributed under the Creative Commons Attribution License, which permits unrestricted use, distribution, and reproduction in any medium, provided the original work is properly cited.

\begin{abstract}
This work develops an advanced quadrature-tracking demodulation technique for coherently demodulating the orthogonal frequency-division multiplexing (OFDM) signal of LTE-A systems. To overcome the fact that traditional coherent demodulators are extremely sensitive to the quadrature imbalance of a system, especially an OFDM system, the proposed architecture uses a novel quadrature phase-locked loop (QPLL) to track simultaneously the in phase (I-phase) and the quadrature phase (Q-phase) of the received signal. This advanced quadrature-tracking demodulator is realized using TSMC $0.18 \mu \mathrm{m}$ CMOS technology and hybrid circuits. Experimental results indicate that the developed quadrature-tracking demodulator, which operates at $2.1 \sim 2.5 \mathrm{GHz}$, can effectively demodulate an 18 Mbps LTE-A signal, even with a 15 degree quadrature imbalance.
\end{abstract}

\section{Introduction}

Most wireless communication systems use coherent demodulation, mainly because the quality of coherent demodulation is much better than that of non-coherent demodulation [1]. Traditional coherent demodulators are based on an RF quadrature demodulator. But the RF coherent circuits are usually complex and power-hungry [2]. To simplify the receiver's circuitry, various phase-locked loop (PLL)-based coherent demodulators are adopted in wireless communication systems. The most well-known PLL-based coherent demodulator has the Costas architecture [1,3-5]. As depicted in Figure 1, this architecture uses a single PLL with two feedback loops. These two feedback loops demodulate the in-phase (I-phase) signal and quadrature-phase (Q-phase) signals, respectively. The demodulated signals are combined, and then tune the voltage-controlled oscillator (VCO) to track the frequency of the carrier signal. However, both the traditional quadrature demodulator and the Costas-coherent demodulator face the problem of quadrature imbalance of $\mathrm{RF}$ signal. Quadrature imbalance of RF signal arises from both the quadrature transmitter and the quadrature receiver. Previous investigations have showed that a slight 2.5 degree quadrature imbalance significantly degrades the demodulation quality of an OFDM signal, which is extensively used in LTE-A systems [6, 7]. Although the conventional Costas-coherent demodulator has two feedback loops for demodulation, the single-VCO design prevents tracking of more than one phase of a received signal.

We [8] previously presented an alternative coherent polar demodulator without the quadrature imbalance problem of receiver. As presented in Figure 2, the received signal is divided into two paths. One of these paths uses injectionlocked oscillators (ILO) to extract the phase-modulated carrier signal and the phase information. The extracted phase-modulated carrier is then mixed with the received signal along another path. The mixing cancels out the phase information of these two input signals, and then the envelope information of the received signal is exported. The baseband processor then recovers the baseband signal from both the demodulated phase and the envelope information. However, the quadrature imbalance that is caused by the RF transmitter remains in the received signal.

To overcome the quadrature imbalance problem, some works directly trimming or adjusting their RF circuits [9]. 


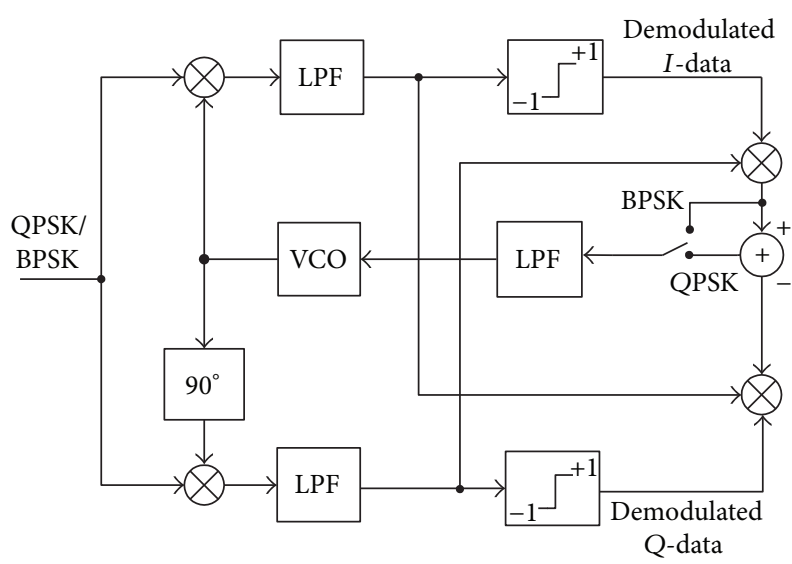

Figure 1: Traditional Costas demodulator.

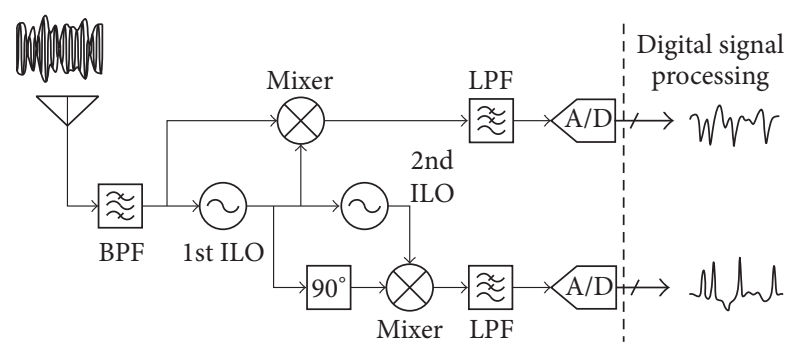

FIgURE 2: Polar demodulator.

However, these are impractical. Another solution is basedon digital-signal process (DSP) technique. [10] and [11] respectively uses the pilot signal and a special tone to train the DSP in receiver to find out and then correct the quadrature imbalance. $[9,12]$ utilize adaptive algorithms to estimate the quadrature error and then compensate demodulated signal. Although these adaptive algorithms theoretically can reduce the quadrature imbalance to less than 1 degree, they take a very long computation time with about $10^{5}$ iterations. To speed up the tracking process, this work proposes a novel quadrature-tracking demodulator which can real-time track the quadrature error.

\section{Quadrature-Tracking Demodulator}

To eliminate the extreme sensitivity of traditional coherent demodulation to the quadrature phase imbalance, this work proposes a novel quadrature phase-locked loop (QPLL)based coherent demodulator. As depicted in Figure 3(a), the proposed QPLL is based mainly on two identical PLLs with a channel-preset frequency synthesizer. Unlike the traditional Costas-coherent demodulator which uses a single-ended VCO, the QPLL-based demodulator utilizes a novel quadrature voltage-controlled oscillator (QVCO). As presented in Figure 3(b), the QVCO is formed by cross-coupling two identical differential VCOs. The tuning ports of these two VCOs are independent of each other, rather than connected. One performs I-phase tracking in the QPLL while the other performs Q-phase tracking. Under the initial condition of demodulation, the two tuning ports of the QVCO are shorted to make the QPLL act as a single PLL to lock the carrier frequency of the received signal. Since the effective detection range of a mixer-based phase detector is limited by \pm 90 degree [13], as depicted in Figure 4, an additional channelpreset frequency synthesizer is required. The additional frequency synthesizer uses an all-digital phase-frequency detector (PFD) to detect a large phase variance of up to \pm 360 degree. Therefore, the QPLL can track both the frequency and the phase of the received signal. After the frequency of the received signal has been locked, the channel-preset frequency synthesizer is turned off to save power and the two VCO tuning ports are disconnected, as presented in Figure 5. The QPLL can then track in real time and demodulate both the $I$-phase and the Q-phase of the received signal. According to PLL theory, a PLL-based demodulator attenuates the demodulated signal within the loop bandwidth of the PLL [13]. Therefore, the proposed architecture is especially suited to OFDM systems because the DC-subcarrier of the OFDM signal, as depicted in Figure 6, is not used in the LTE-A system, to mitigate the DC-offset problem [14]. Therefore, the proposed advanced QPLL-based demodulation technique can coherently demodulate the OFDM signal without attenuation if the loop bandwidth of the PLL is designed to be less than the sub-carrier space.

\section{System Analysis}

To analyze the proposed quadrature-tracking demodulator in the time domain, the received signal is assumed to be

$$
\begin{aligned}
r(t)= & I(t) \cos \left[\left(\omega_{0}+\Delta \omega\right) t+\theta_{I}\right] \\
& +Q(t) \sin \left[\left(\omega_{0}+\Delta \omega\right) t+\theta_{Q}\right],
\end{aligned}
$$

where $I(t)$ and $Q(t)$ denote baseband signals, and $\Delta \omega$ is the frequency error; $\theta_{I}$ and $\theta_{Q}$ are the phase errors of the $I$ phase signal and the $Q$-phase signal, respectively. After downmixing, the signal at nodes $A$ and $B$ in the circuit that is displayed in Figure 5 is derived as

$$
\begin{aligned}
A(t)= & \frac{I(t)}{2} \sin \left[\left(2 \omega_{0}+\Delta \omega\right) t+\theta_{I}+\theta_{I}^{\prime}\right] \\
& +\frac{I(t)}{2} \sin \left(\Delta \omega t+\Delta \theta_{I}\right) \\
& +\frac{Q(t)}{2} \cos \left(\Delta \omega t+\theta_{\mathrm{Q}}-\theta_{I}^{\prime}\right) \\
& -\frac{Q(t)}{2} \cos \left[\left(2 \omega_{0}+\Delta \omega\right) t+\theta_{I}^{\prime}+\theta_{\mathrm{Q}}\right] \\
B(t)= & \frac{I(t)}{2} \cos \left[\left(2 \omega_{0}+\Delta \omega\right) t+\theta_{I}+\theta_{I}^{\prime}\right] \\
& +\frac{I(t)}{2} \cos \left(\Delta \omega t+\theta_{I}-\theta_{\mathrm{Q}}^{\prime}\right) \\
& +\frac{Q(t)}{2} \sin \left(\Delta \omega t+\Delta \theta_{\mathrm{Q}}\right) \\
& +\frac{Q(t)}{2} \sin \left[\left(2 \omega_{0}+\Delta \omega\right) t+\theta_{\mathrm{Q}}^{\prime}+\theta_{\mathrm{Q}}\right],
\end{aligned}
$$




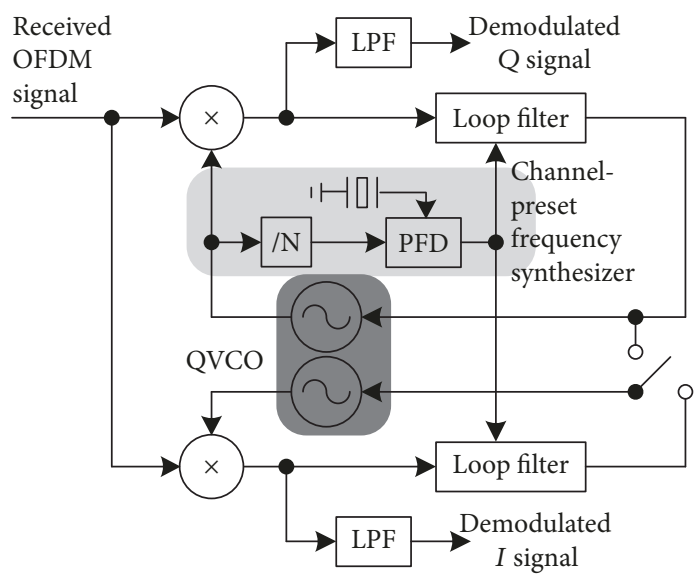

(a)

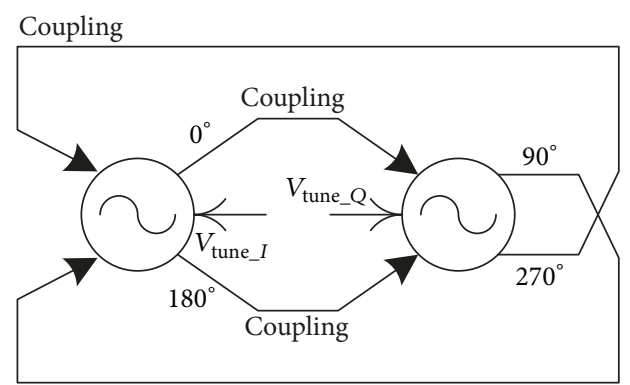

(b)

Figure 3: Proposed (a) quadrature-tracking demodulator, and (b) QVCO.

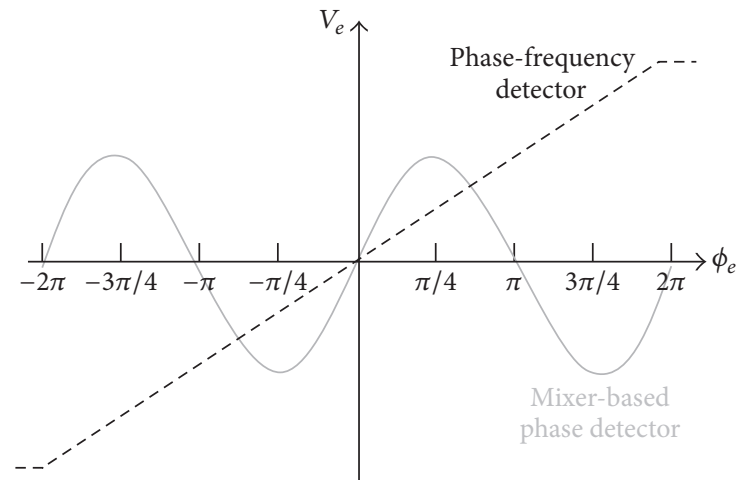

FIGURE 4: Effective phase-detection range of a mixer-based phase detector.

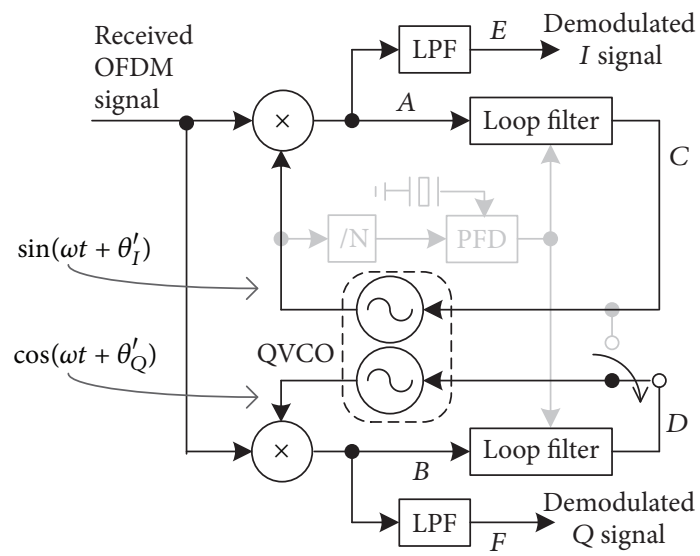

FIGURE 5: Proposed demodulator under quadrature-tracking and demodulating conditions.

where

$$
\begin{gathered}
\Delta \theta_{I}=\theta_{I}-\theta_{I}^{\prime}, \\
\Delta \theta_{\mathrm{Q}}=\theta_{\mathrm{Q}}-\theta_{\mathrm{Q}}^{\prime}
\end{gathered}
$$

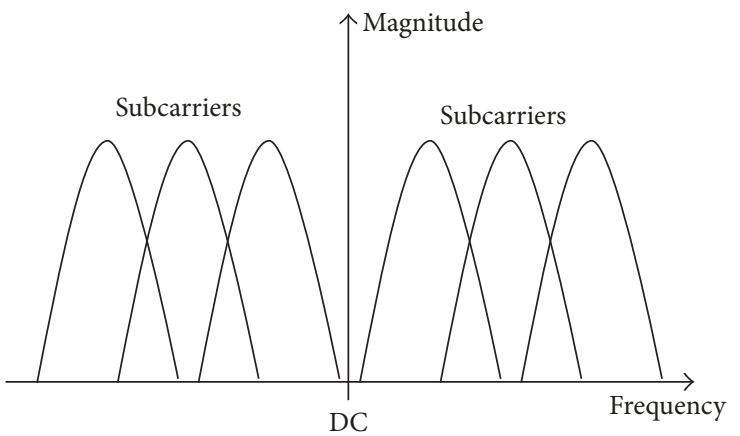

FIGURE 6: Sub-carrier spectrums of OFDM signal in LTE-A system.

are the I-phase and Q-phase phase errors between the transmitter and the receiver. Since the loop bandwidth of the PLL must be narrower than the sub-carrier space of the OFDM signal, the signal at nodes $C$ and $D$ of the circuit in Figure 5 are derived as

$$
\begin{array}{ll}
C(t) \cong \frac{I(t)\left(\Delta \omega t+\Delta \theta_{I}\right)}{2}, & \text { for }\left|\Delta \omega t+\Delta \theta_{I}\right|<\frac{\pi}{4} \\
D(t) \cong \frac{Q(t)\left(\Delta \omega t+\Delta \theta_{Q}\right)}{2}, & \text { for }\left|\Delta \omega t+\Delta \theta_{I}\right|<\frac{\pi}{4}
\end{array}
$$

According to PLL theory, the negative-feedback mechanism makes $\Delta \omega, \Delta \theta_{I}$, and $\Delta \theta_{Q}$ equal zero. Under these conditions, the QPLL-based demodulator locks both the frequency and the phase of the received RF signal.

To extract the demodulated baseband signals from the QPLL-based demodulator, two additional low-pass filters are used at the output of phase detectors. The cut-off frequency of the filter must exceed the top of the frequency band of the baseband signal. The signals at nodes $E$ and $F$, shown in Figure 5 are derived as

$$
\begin{aligned}
E(t)= & \frac{Q(t)}{2} \cos \left(\Delta \omega t+\Delta \theta_{Q}\right) \\
& -\frac{I(t)}{2} \sin \left(\Delta \omega t+\Delta \theta_{I}\right),
\end{aligned}
$$




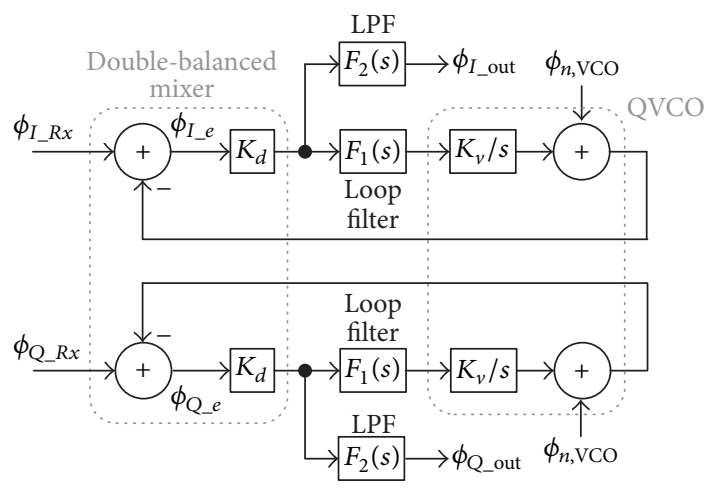

FIGURE 7: Linear phase model of proposed quadrature-tracking demodulator.

$$
\begin{aligned}
F(t)= & \frac{I(t)}{2} \cos \left(\Delta \omega t+\Delta \theta_{I}\right) \\
& +\frac{Q(t)}{2} \sin \left(\Delta \omega t+\Delta \theta_{Q}\right)
\end{aligned}
$$

Since $\Delta \omega, \Delta \theta_{I}$, and $\Delta \theta_{Q}$ are zero when the PLL locks the frequency and phase of the received signal, the demodulated Q-phase signal and $I$-phase signal are found as

$$
\begin{aligned}
& E(t) \cong \frac{Q(t)}{2} \\
& F(t) \cong \frac{I(t)}{2}
\end{aligned}
$$

Equations (6) indicate that the novel demodulator can effectively demodulate the received signal without distortions, which would otherwise be caused by the quadratureimbalance.

To further analyze the frequency response of the proposed demodulator, a frequency domain linear model is developed, as presented in Figure 7. $F_{1}(s)$ and $F_{2}(s)$ represent the frequency responses of the loop filters. $K_{v}$ and $K_{d}$ represent the sensitivities of the QVCO and mixer-based phase detector, respectively. $\phi_{n, \mathrm{VCO}}$ represents the phase noise of the QVCO.

$$
\begin{gathered}
\phi_{I \_x}(s)=\phi_{I}(s)+\phi_{I_{-} e} \\
\phi_{\mathrm{Q} \_x}(s)=\phi_{\mathrm{Q}}(s)+\phi_{\mathrm{Q} \_e}
\end{gathered}
$$

represent the $I$-phase and $Q$-phase of the received signal, respectively, where $\phi_{I_{\_} e}$ and $\phi_{\mathrm{Q}_{\_} e}$ are the quadrature phase errors. $\phi_{I_{-} \text {out }}$ and $\phi_{\text {Q_out }}$ denote the phases of the demodulated signals, respectively, and are given by

$$
\begin{aligned}
\phi_{I_{-} \mathrm{out}}(s) & =\left[\phi_{I}(s)+\phi_{I_{-} e}-\phi_{n, \mathrm{VCO}}(s)\right] F_{2}(s) H_{e}(s) \\
& =\left[\phi_{I}(s)-\phi_{n, \mathrm{VCO}}(s)\right] F_{2}(s) H_{e}(s)+\phi_{I_{-} e}(s) \\
\phi_{\mathrm{Q}_{\mathrm{out}}}(s) & =\left[\phi_{\mathrm{Q}}(s)+\phi_{\mathrm{Q}_{e}}-\phi_{n, \mathrm{VCO}}(s)\right] F_{2}(s) H_{e}(s) \\
& =\left[\phi_{I}(s)-\phi_{n, \mathrm{VCO}}(s)\right] F_{2}(s) H_{e}(s)+\phi_{I_{e}}(s),
\end{aligned}
$$

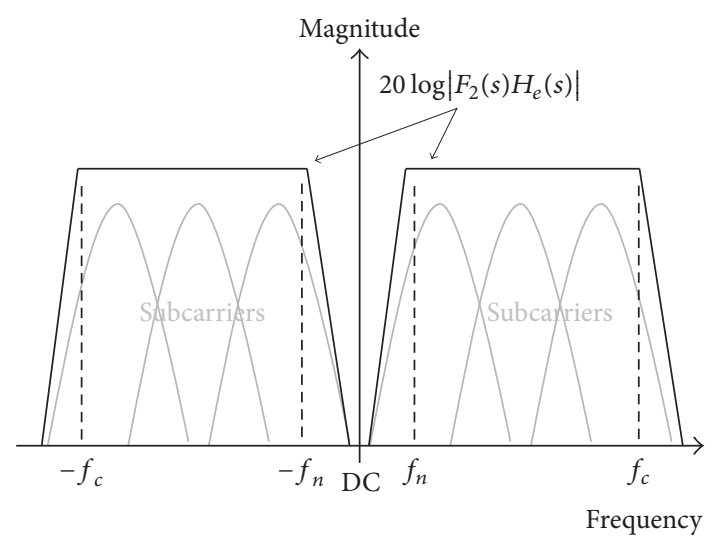

FIGURE 8: Frequency response of proposed quadrature tracking demodulator.

where

$$
H_{e}(s)=\frac{s}{s+K_{v} K_{d} F_{1}(s)}
$$

is the error transfer function of the QPLL, and

$$
\begin{gathered}
\phi_{I_{-} e}(s)=\phi_{I_{-} e} F_{2}(s) H_{e}(s), \\
\phi_{\mathrm{Q}_{e} e}(s)=\phi_{\mathrm{Q}_{-} e} F_{2}(s) H_{e}(s) .
\end{gathered}
$$

According to final value theory, the output quadrature errors $\phi_{I_{-} e}(s)$ and $\phi_{Q_{-} e}(s)$ are zero when the quadrature phase errors $\phi_{I_{-} e}$ and $\phi_{Q_{-} e}$ are constant [15]. Under these conditions, Eqs. (8), can be simplified as

$$
\begin{aligned}
\phi_{I_{-} \mathrm{out}}(s) & =\left[\phi_{I}(s)+\phi_{I_{-} e}-\phi_{n, \mathrm{VCO}}(s)\right] F_{2}(s) H_{e}(s) \\
& =\left[\phi_{I}(s)-\phi_{n, \mathrm{VCO}}(s)\right] F_{2}(s) H_{e}(s), \\
\phi_{\mathrm{Q} \_ \text {out }}(s) & =\left[\phi_{\mathrm{Q}}(s)+\phi_{\mathrm{Q} \_}-\phi_{n, \mathrm{VCO}}(s)\right] F_{2}(s) H_{e}(s) \\
& =\left[\phi_{I}(s)-\phi_{n, \mathrm{VCO}}(s)\right] F_{2}(s) H_{e}(s) .
\end{aligned}
$$

Figure 8 plots the frequency response of $F_{2}(s) H_{e}(s)$, where $f_{n}$ represents the loop bandwidth of the QPLL and $f_{c}$ is the cut-off frequency of the LPF. The $f_{n}$ should be designed to be narrower than the frequency gap between subcarriers to prevent distortion. $f_{c}$ should equal the channel bandwidth of the system to enable channel selection. From the spectrum of the OFDM sub-carriers and the frequency response of the demodulator in Figure 8, the proposed architecture can coherently demodulate the received OFDM signal without any distortion. Moreover, the phase noise $\phi_{n \text {,VCO }}$ from the QVCO can be effectively suppressed by the demodulator in the loop bandwidth $f_{n}$, too.

Figures 9(a) and 9(b) presents the system-level simulation result of demodulated spectrum of traditional quadrature demodulator and the proposed quadrature tracking demodulator, respectively. In the simulation, the modulation type is set to be OFDM with 64 sub-carriers. These results show that the demodulated spectrum of traditional quadrature demodulator is significantly degraded as the quadrature 


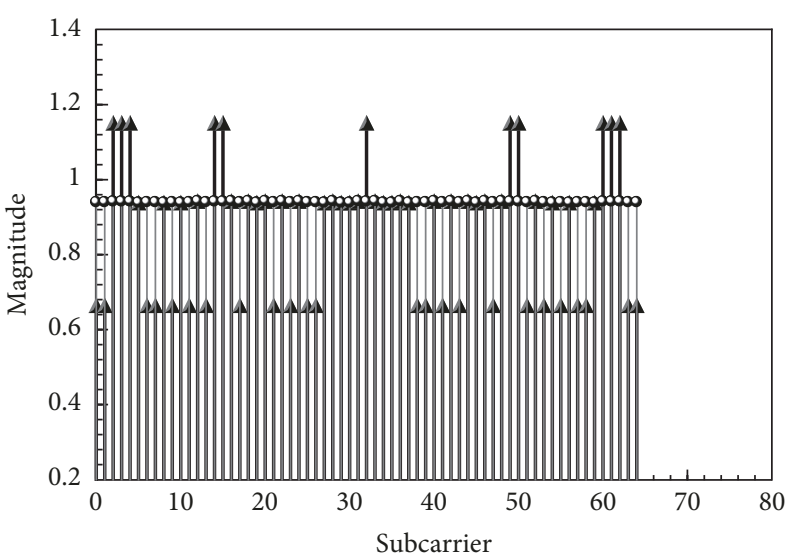

- 0 deg quadrature imbalance

^ 15 deg quadrature imbalance

(a)

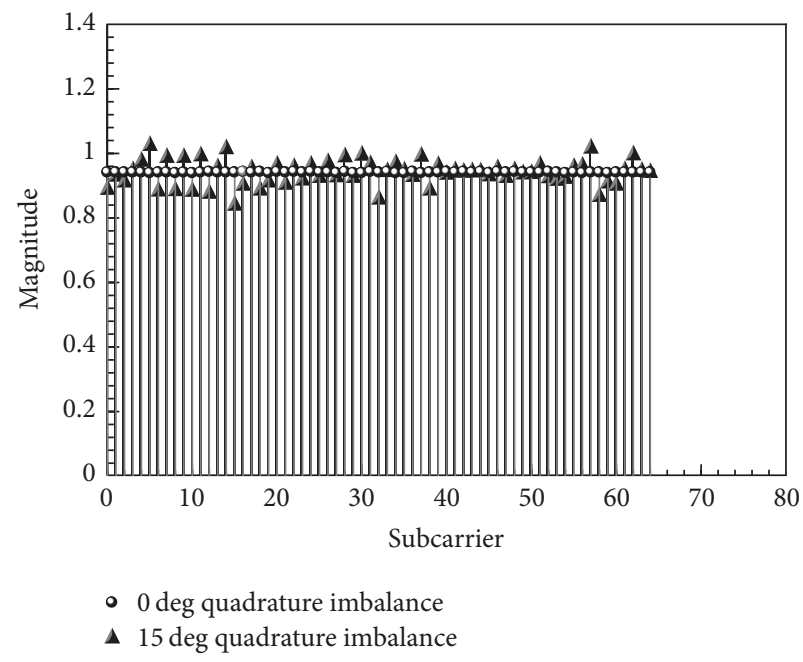

(b)

FIGURE 9: Demodulated spectrum of (a) traditional quadrature demodulator and (b) proposed quadrature tracking demodulator.

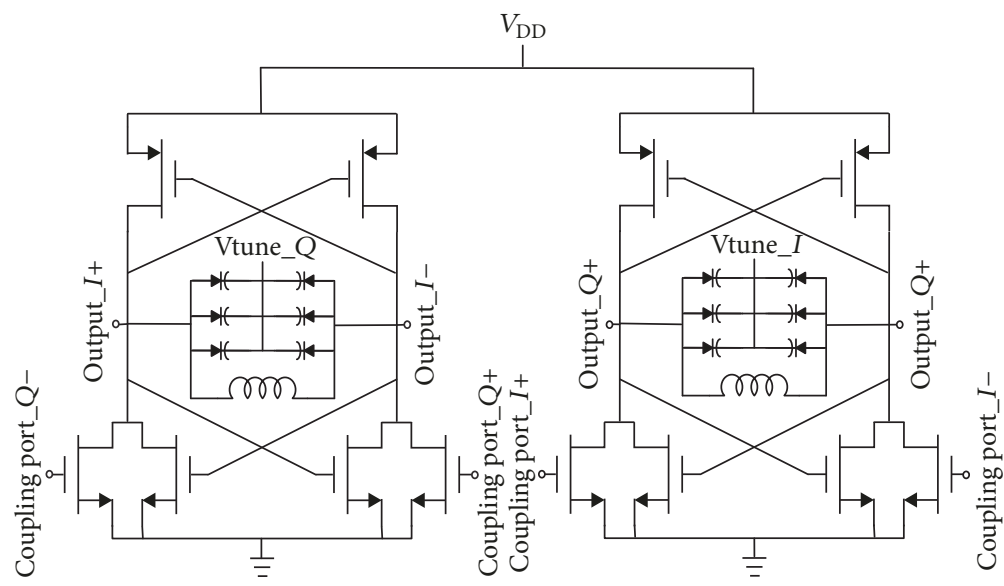

(a)

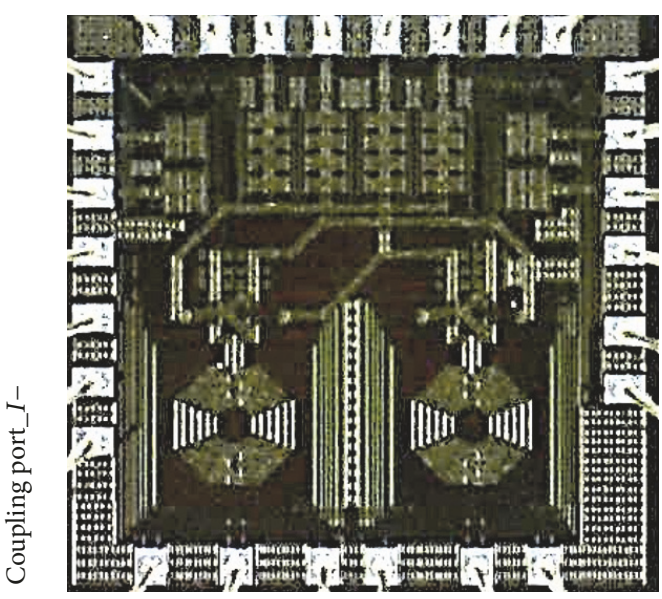

(b)

FIgURE 10: (a) Circuit and (b) CMOS chip of the QVCO.

imbalance increasing from 0 degree to 15 degree. Under the same condition, the demodulated spectrum of the proposed quadrature tracking demodulator shows a very slight decline.

\section{Experimental Results}

The QVCO of the proposed demodulator was implemented using TSMC $0.18 \mu \mathrm{m}$ CMOS technology. Figure 10(a) shows the circuit design of the CMOS QVCO. Generally, a VCO with internal NMOS cross-coupled pairs has a wider operating range but a poorer phase noise performance than the one with internal PMOS cross-coupled pairs [18]. Based on consideration of both the phase noise performance and operating range, the internal complementary crosscoupled pair is used. To realize quadrature outputs, external cross-coupling between two identical differential VCOs are required so four additional NMOS are designed to be parallel to the original NMOS cross-coupled pair. The outputs of two identical differential VCOs then cross-couple to each other via the gates of the additional NMOSs. These internal and external couplings of the VCOs force the four outputs signal quadrature each other. Since the QPLL must separately track the $I$-phase and the $Q$-phase of the received signal, the QVCO is designed to have two tuning ports, as presented in Figure 10(a). This architecture enables the quadrature of the QVCO to be slightly adjusted using the two independent tuning ports.

Figure 10(b) displays the implemented CMOS QVCO chip. The measured power consumption is less than $8.9 \mathrm{~mW}$. The operating range and output power of the CMOS QVCO are measured and presented in Figure 11(a). The CMOS QVCO can operate from 2.1 to $2.5 \mathrm{GHz}$ with a mean output power of around $2 \mathrm{dBm}$, which is sufficiently high to drive passive double-balance mixers for phase detection. The measured output power of the QVCO also exhibits very 


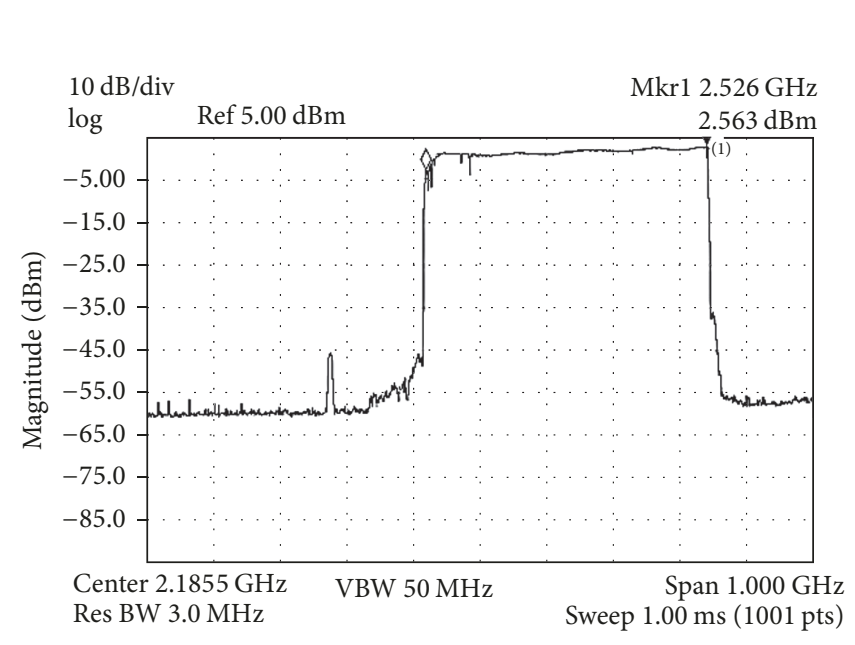

(a)
Phase noise $(\mathrm{dBc} / \mathrm{Hz})$

RF atten $5 \mathrm{~dB}$

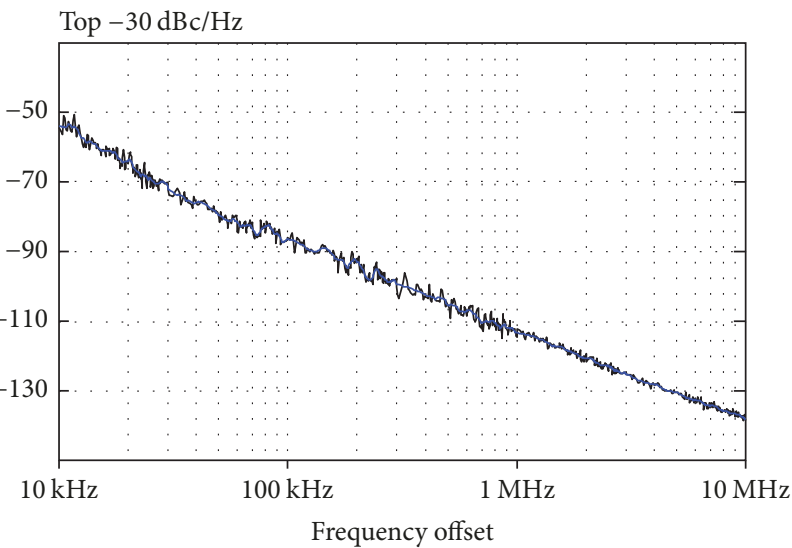

(b)

FIGURE 11: Measured (a) operating range and output power, and (b) phase noise of CMOS QVCO of demodulator.

TAble 1: Performance Comparison of $2.4 \mathrm{GHz}$ QVCOs.

\begin{tabular}{lccc}
\hline & This work & {$[16]$} & {$[17]$} \\
\hline Process & $0.18 \mu \mathrm{m}$ CMOS & $0.18 \mu \mathrm{m}$ CMOS & $0.18 \mu \mathrm{m}$ CMOS \\
Supply voltage $(\mathrm{V})$ & 1.4 & 0.6 & 1.8 \\
Power dissipation $(\mathrm{mW})$ & 5.5 & 2.4 & 14.8 \\
Frequency (GHz) & 2.4 & 9.5 & 2.4 \\
Fractional bandwidth (\%) & 17.5 & -10 & 26.3 \\
Output power (dBm) & 2 & $-110 \sim-104$ & 0 \\
Phase noise @1 MHz (dBc/Hz) & -113 & 2.64 & -112 \\
Quadrature error (Degree) & 6.9 & 166.4 & 5 \\
FoM @1 MHz & 173.2 & & 168.3 \\
\hline
\end{tabular}

good flatness of better than $2 \mathrm{~dB}$ over the wide operating range. Figure 11(b) plots the measured phase noise of the CMOS QVCO. The phase noise is lower than $-113 \mathrm{dBc} / \mathrm{Hz}$ and $-137 \mathrm{dBc} / \mathrm{Hz}$ at $1 \mathrm{MHz}$ and $10 \mathrm{MHz}$ offset frequency, respectively. Table 1 shows the performance comparison between $2.4 \mathrm{GHz}$ CMOS QVCOs, where the figure of merit (FoM) is given by [19]

$$
\text { FoM }=-\mathrm{L}(\Delta \omega)+20 \log \left(\frac{\omega_{0}}{\Delta \omega}\right)-10 \log \left(\frac{P_{\text {diss }}}{1 m W}\right) .
$$

The comparison shows that the implemented QVCO achieves a lowest phase noise and power consumption, and consequently results in a better FoM over the other QVCOs.

The proposed quadrature-tracking demodulator is implemented with the designed CMOS QVCO, as presented in Figure 12. The channel-preset frequency synthesizer of the demodulator is implemented using a consumer IC PE3336, produced by Peregrine. The phase detectors are the ZX05C42 S+ model from Mini-Circuits. The LPFs are the SLP$10.7+$ model, also from Mini-Circuits, and have a $3 \mathrm{~dB}$ bandwidth of $14 \mathrm{MHz}$. The loop bandwidth of the QPLL is designed to be $10 \mathrm{kHz}$. The received LTE-A signal of the receiver is generated using a Keysight MXA vector signal

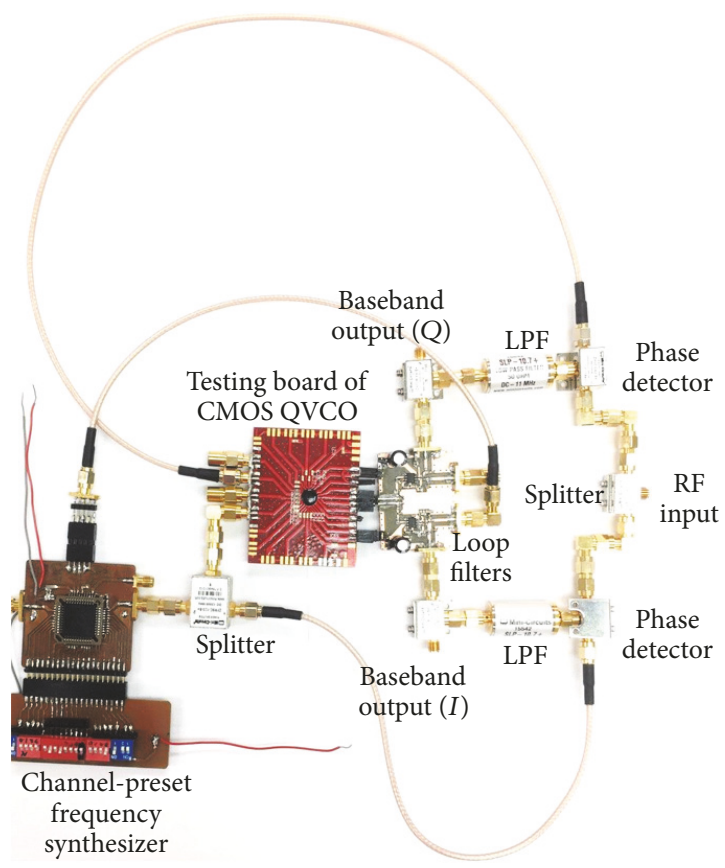

FIGURE 12: Implemented quadrature-tracking de-modulator. 


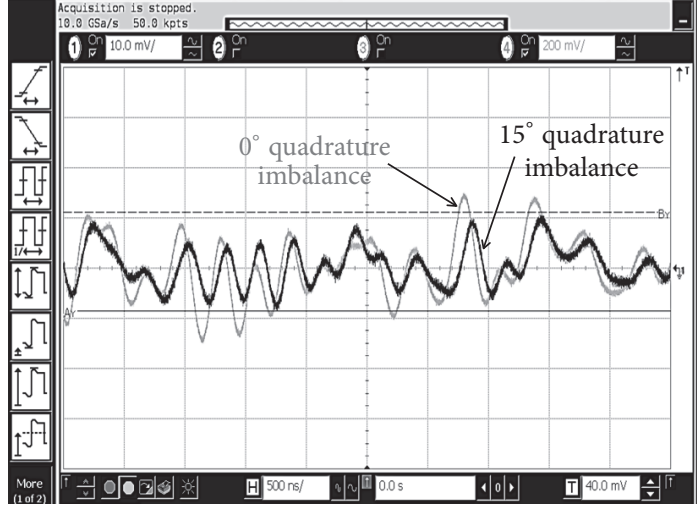

(a)

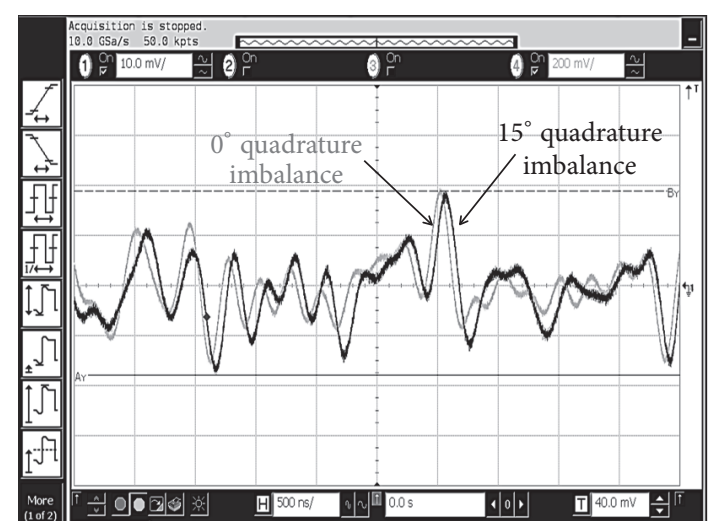

(b)

Figure 13: Demodulated (a) I-phase and (b) Q-phase baseband waveforms when received LTE-A signal has a quadrature imbalance of $15^{\circ}$.

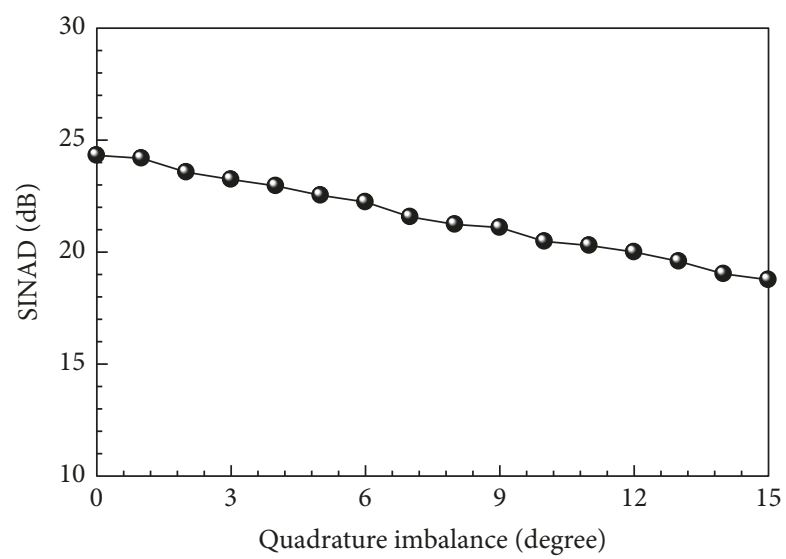

FIGURE 14: Measured SINAD of the implemented quadraturetracking demodulator.

generator (VSG) with Keysight Signal Studio. The data rate of the LTE-A signal is set to be $18 \mathrm{Mbps}$. The sub-carrier space is set to $156.25 \mathrm{kHz}$, which greatly exceeds the $10 \mathrm{kHz}$ loop bandwidth of the QPLL.

Figures 13(a) and 13(b) presents the demodulated $I$ phase and Q-phase waveforms, respectively. To confirm the quadrature-tracking performance of the implemented demodulator, the quadrature-imbalance of the LTE-A signal is manually adjusted in the signal studio. As the quadratureimbalance is increased from 0 degree to 15 degree, both the $I$-phase and the $Q$-phase waveforms are degraded slightly revealing that the implemented quadrature-tracking demodulator can effectively demodulate the LTE-A signal even if the signal has a $15^{\circ}$ quadrature-imbalance.

Figure 14 shows the measured signal to noise and distortion ratio (SINAD) degradation of the implemented demodulator as a function of quadrature imbalance. Comparing with the well-known signal to noise ratio (SNR), SINAD further considers the combining effect of both noise and distortion.
Therefore, the SINAD is usually used to evaluate performance of RF receivers [20]. SINAD is defined as

$$
\begin{aligned}
\text { SINAD } & =10 \log \frac{P_{S}+P_{N}+P_{D}}{P_{N}+P_{D}} \\
& =10 \log \frac{P_{\mathrm{DS}}}{P_{N}+P_{D}}(\mathrm{~dB}),
\end{aligned}
$$

where $P_{S}, P_{\mathrm{DS}}, P_{N}$, and $P_{D}$ represents the power of the ideal signal, distorted signal, noise, and distortion, respectively. The measured results show that the SINAD slightly degrade as the quadrature imbalance increase from 0 degree to 15 degree. As the quadrature imbalance is 15 degree, the SINAD of the implemented quadrature-tracking demodulator is about $18.761 \mathrm{~dB}$ which is acceptable for most wireless communication applications.

\section{Conclusion}

In this work, a novel quadrature tracking demodulator for LTE-A applications was implemented. The $2.1 \sim 2.5 \mathrm{GHz}$ QPLL-based demodulator can effectively demodulate an $18 \mathrm{Mbps}$ LTE-A signal with a quadrature-imbalance of up to 15 degrees. This remarkable quadrature-tracking ability makes the novel demodulator well suited to LTE-A systems or even more advanced communication systems.

\section{Conflicts of Interest}

The authors declare that there is no conflicts of interest regarding the publication of this paper

\section{Acknowledgments}

The authors would like to thank the Ministry of Science and Technology (101-2221-E-327-029) for providing research funding. The authors would also like to thank the National Chip Implementation Center, Hsinchu, Taiwan, for providing the CMOS foundry service. 


\section{References}

[1] S. Haykin and M. Moher, Communication Systems, Wily, USA, 5th edition, 2009.

[2] B. Razavi and RF. Microelectronics, Prentice Hall Inc.

[3] G. L. Do, "An ultra-fast carrier recovery versus traditional synchronizers," IEEE Transactions on Broadcasting, vol. 42, no. 1, pp. 42-49, 1996.

[4] J. P. Costas, "Synchronous communications," Proceedings of the IEEE, vol. 90, no. 8, pp. 1461-1466, 2002.

[5] S.-J. Huang, Y.-C. Yeh, H. Wang, P.-N. Chen, and J. Lee, "Wband BPSK and QPSK transceivers with Costas-loop carrier recovery in 65-nm CMOS technology," IEEE Journal of SolidState Circuits, vol. 46, no. 12, pp. 3033-3046, 2011.

[6] M. Buchholz, A. Schuchert, and R. Hasholzner, "Effects of tuner IQ imbalance on multicarrier-modulation systems," in Proceedings of the ICCDCS 2000: 3rd IEEE International Conference on Devices, Circuits and Systems, pp. T65/1-T65/6, March 2000.

[7] M. Rumney, LTE and the Evolution to 4G Wireless: Design and Measurement Challenges, John Wiley \& Sons, West Sussex, UK, 2013.

[8] C.-T. Chen, C.-H. Hsiao, T.-S. Horng, K.-C. Peng, and C.-J. Li, "Cognitive polar receiver using two injection-locked oscillator stages," IEEE Transactions on Microwave Theory and Techniques, vol. 59, no. 12, pp. 3484-3493, 2011.

[9] R. Cherukuri and P. T. Balsara, "Code-aided adaptive decorrelator for IQ imbalance compensation in iterative receivers for flat fading channels," in Proceedings of the Military Communications Conference, MILCOM 2007, USA, October 2007.

[10] L. Giugno, V. Lottici, and M. Luise, "Efficient compensation of I/Q phase imbalance for digital receivers," in Proceedings of the 2005 IEEE International Conference on Communications, ICC 2005, pp. 2462-2466, May 2005.

[11] C.-H. Hsu, C.-F. Wu, and C.-K. Wang, "FPGA prototype for WLAN OFDM baseband with STPE of I/Q mismatch self calibration algorithm," in Proceedings of the 1st IEEE Asian SolidState Circuits Conference, ASSCC 2005, pp. 509-512, Taiwan, November 2005.

[12] G. Vallant, M. Epp, W. Schlecker, U. Schneider, L. Anttila, and M. Valkama, "Analog IQ impairments in Zero-IF radar receivers: Analysis, measurements and digital compensation," in Proceedings of the IEEE International Instrumentation and Measurement Technology Conference, I2MTC 2012, pp. 17031707, Austria, May 2012.

[13] K. C. Peng, Modulated Frequency Synthesizers: Design for Wideband and High Efficiency Modulation, VDM Verlag, Germany, 2009.

[14] M. Rumney, LTE and the Evolution to 4G Wireless - Design and Measurement Challenges, Second Edition, John Wiley \& Sons, West Sussex, UK, 2008.

[15] K.-C. Peng, C.-H. Lee, C.-H. Chen, and T.-S. Horng, "Enhancement of frequency synthesizer operating range using a novel frequency-offset technique for LTE-a and CR applications," IEEE Transactions on Microwave Theory and Techniques, vol. 61, no. 3, pp. 1215-1223, 2013.

[16] C. T. Lu, H. H. Hsieh, and L. H. Lu, "A low-power quadrature VCO and its application to a $0.6-\mathrm{V}$ 2.4-GHz PLL," IEEE Transactions on Circuits and Systems I: Regular Papers, vol. 57, no. 4, pp. 793-802, 2010.
[17] S. Shin, K. Lee, and S. M. Kang, "low-power $2.4 \mathrm{GHz}$ CMOS frequency synthesizer with differentially controlled MOS varactors," in Proceedings of the 2006 IEEE International Symposium on Circuits and Systems, pp. 553-556, Island of Kos, Greece.

[18] K. C. Peng and C. H. Lee, "A 5 GHz CMOS quadrature VCO with precise quadrature phase," in Proceedings of the 2012 Asia-Pacific Microwave Conference, APMC 2012, pp. 1211-1213, Taiwan, December 2012.

[19] K. C. Peng and T. S. Horng, "Optimization of phase noise in a 2.3 - $3.5 \mathrm{GHz}$ voltage-controlled oscillator using the impedance locus," in Progress in Electromagnetics Research Symposium Dig, pp. 913-917, 2012.

[20] Wikipedia contributors, "SINAD," Wikipedia, The Free Encyclopedia, Available: https://en.wikipedia.org/wiki/SINAD. 


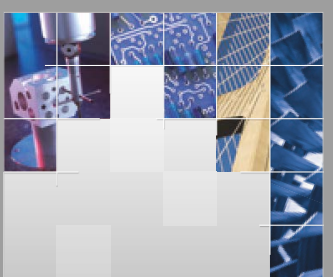

\section{Enfincering}
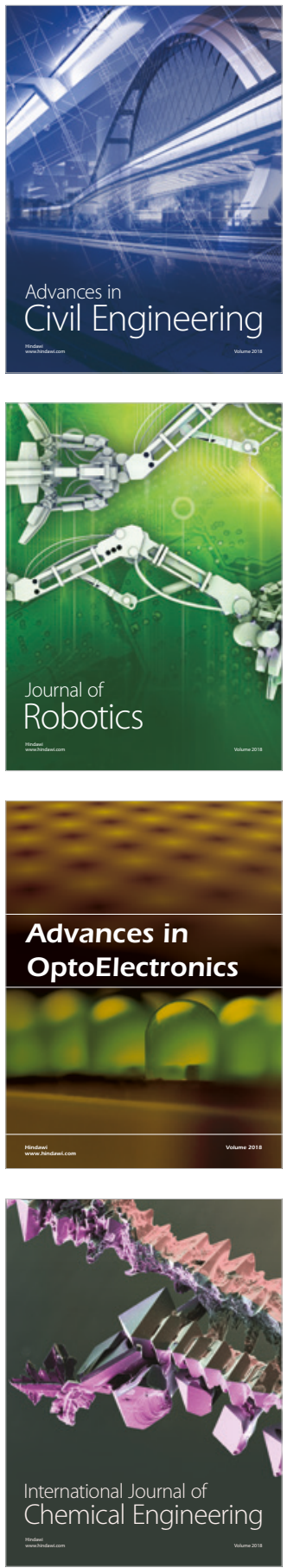

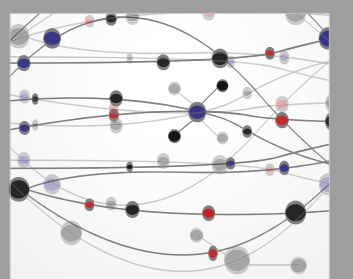

\section{Rotating \\ Machinery}

The Scientific World Journal

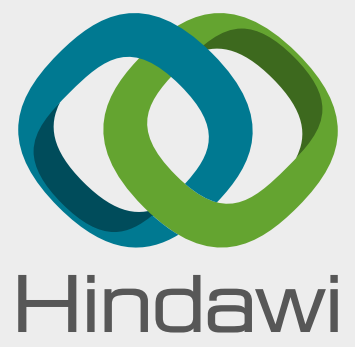

Submit your manuscripts at

www.hindawi.com
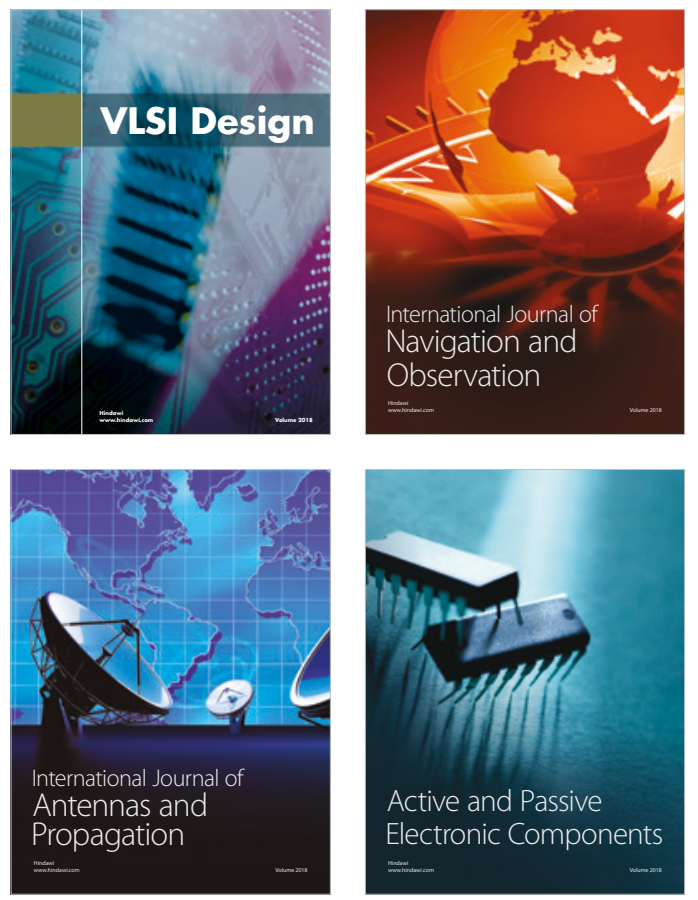
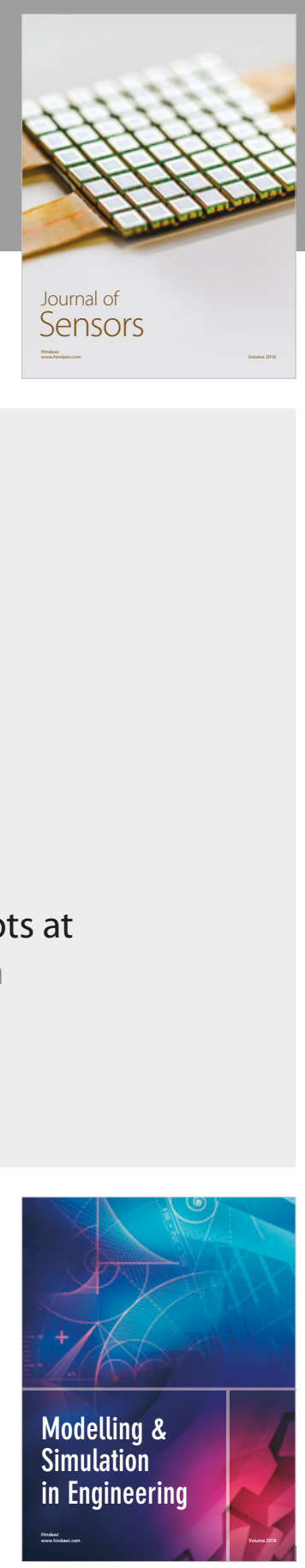

\section{Advances \\ Multimedia}
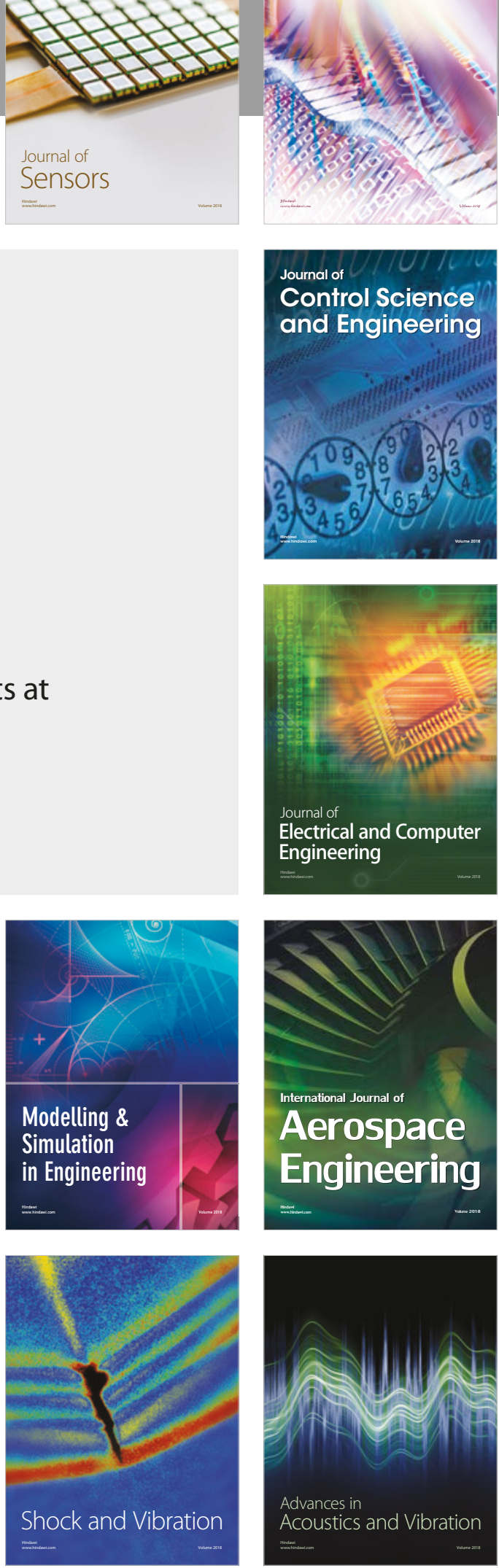